\title{
A Self Monitoring and Analyzing System for Solar Power Station using IoT and Data Mining Algorithms
}

\author{
Subarna Shakya, \\ Professor, \\ Department of Electronics and Computer Engineering, \\ Central Campus, Institute of Engineering, Pulchowk, \\ Tribhuvan University, \\ Pulchowk, Lalitpur, Nepal. \\ Email: drss@ioe.edu.np
}

Abstract: Renewable energy sources are gaining a significant research attention due to their economical and sustainable characteristics. In particular, solar power stations are considered as one of the renewable energy systems that may be used in different locations since it requires a lower installation cost and maintenance than conventional systems, despite the fact that they require less area. In most of the small generating stations, space occupancy is controlled by placing the equipment on an open terrace. However, for large-scale power generating stations, acres of land are required for installation. Human employers face a challenging task in maintaining such a large area of power station. Through IoT and data mining techniques, the proposed algorithm would aid human employers in detecting the regularity of power generation and failure or defective regions in solar power systems. This allows performing a quick action for the fault rectification process, resulting in increased generating station efficiency.

Keywords: Solar panel maintenance, maximum power generation, IoT, data mining, solar panel.

\section{Introduction}

Maintenance is required for all the modern systems. Furthermore, the preventive and breakdown maintenance are two types of maintenance. When compared to electrical or electronic systems, the mechanical systems require a lot of preventive maintenance. The scheduled maintenance, routine maintenance, and predictive maintenance are all remaining as the elements of preventative maintenance [1]. The electrical and electronics systems like 
Journal of Soft Computing Paradigm (JSCP) (2021)

Vol.03/ No.02

Pages: 96-109

http://irojournals.com/jscp/

DOI: https://doi.org/10.36548/jscp.2021.2.004

television, mobile phones, fan, lights etc., are mostly does not require any planned or routine maintenance. However, the defective or breakdown of an electrical system can be predicted by monitoring its performance in a continuous way. Therefore in most of the electrical operations, the systems are equipped with an ammeter and voltmeter for measuring its load conditions [2].

Monitoring a system with human effort is a time consuming and difficult task for an unskilled person in such particular field. Therefore, the monitoring process can be simplified through implementation of several sensors and its peripheral units. Mostly, the sensors are small and tiny in size with the ability to work on any climatic condition with a lesser power requirement. Therefore, there is no worry about the heating and other limitations in the sensor modules [3, 4]. The sensor outputs are mostly numerical and binary in nature so it is mandatory for a human to analyze the readings observed from the sensors. Due to development of several computer algorithms, the human effort on analyzing the observed readings are minimized by setting up a threshold value for determining the emergency or immediate action requirements [5]. At present, the algorithms are widely implemented on such sensor based monitoring systems to take several predetermined actions specified by the humans for avoiding delay in the maintenance operations. Therefore, the presence of damage and faults are reduced to its extent [6].

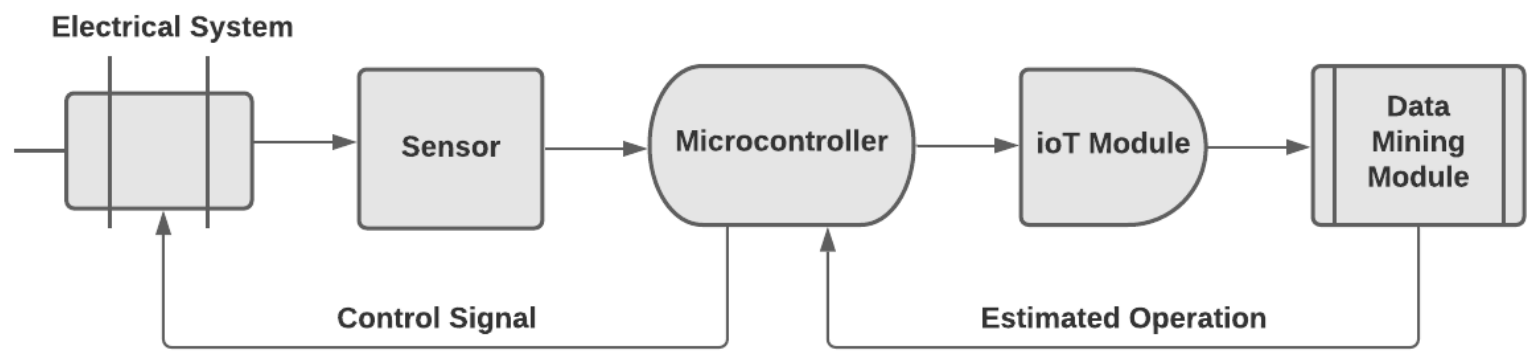

Figure 1. Architecture of IoT based Control Systems

The Internet of Things [IoT] system does not require a huge wiring connection for monitoring any system. The IoT systems also have the ability to transfer the measured readings through wireless connections along with the help of Bluetooth, Wi-Fi and other server modules. Therefore, the installation time, cost and space requirement are comparatively minimal in IoT 
Journal of Soft Computing Paradigm (JSCP) (2021)

Vol.03/ No.02

Pages: 96-109

http://irojournals.com/jscp/

DOI: https://doi.org/10.36548/jscp.2021.2.004

operations and it makes the IoT system to be adaptable for several types of applications. Data that are collected through the IoT sensors can be embedded on any kind of medium for analysis purpose. The developed data mining algorithms based on neural network architectures are having the ability to analyze such readings in the cloud architecture. Therefore, the space requirement for installing a huge hardware setup at the base stating is reduced. The computed information are mostly can be monitored through a regular pc system or an android device. Figure 1 represents the architecture of a monitoring system based on IoT and data mining algorithms.

The difficulty in developing such systems is identification of appropriate sensors and peripheral components. The peripheral components also include the identification of an appropriate neural network algorithm for analysis because there are several type of neural network architectures are available to provide different range of accuracy over the same analysis process depending on its nature. The accuracy of the prediction process is always dependent upon the training process employed in the neural network algorithms. The number of data utilized for the training process will also have the impact over the prediction or estimation analysis. It is becoming a limitation in employing the neural network algorithm on several applications.

\section{Related Work}

The performances of solar power systems are increasing day by day due to the technical advancements of several electronic components. The solar power systems are broadly classified into two types as grid connected systems and standalone systems. The grid connected systems are mostly employed on mass production of electricity and the standalone systems are also employed widely for non-commercial purposes. The grid connected systems usually don't require any battery or power bank modules for storing the generated power for the later utilization. However, the standalone systems are mostly equipped with a storage module for saving the produced energy [7]. While implementing solar power systems with storage modules, it is always must to have a supporting segment for estimating the storage power utilization. A segment based on Bayesian approach [8] was developed to monitor the remaining energy in a lead acid battery. The estimation of remaining energy can be also found for any kind of batteries. 
Journal of Soft Computing Paradigm (JSCP) (2021)

Vol.03/ No.02

Pages: 96-109

http://irojournals.com/jscp/

DOI: https://doi.org/10.36548/jscp.2021.2.004

A support vector regression [9] based technique was also developed to find the balance useful energy on lithium ion batteries. Here the support vector regression model is fused with a particle filter for analyzing the multistep ahead estimation.

Identification and rectification of faults in solar power system is a challenging task nowadays due to its mass space occupancy. Generally monitoring process of grid and transmission system requires a few quantities of reliable sensors for estimating the current and energy flow in a reliable manner. Sensors connected with microcontrollers are having an extra capability to do several actions during the emergency conditions. A hybrid system [10] was developed to do such emergency actions along with the transmission of emergency signal to the authorized base station. To enable such emergency signal to the base station, a GSM module will be kept connected to the microcontroller for data transmission. In the recent years, the monitoring systems are merged with a machine learning approach for analyzing the fault types and its characteristics. The major challenge in employing such intelligent systems is their limited memory and computational capability. Therefore such systems are kept in a base station for having large memory modules and peripheral equipment. In order to catch up the energy changes from the transmission lines the systems are employed with an IoT sensor for transmitting the measured signal to the base station. A similar kind of technique was developed [11] with the help of SVM and fuzzy deep neural network for classifying the faulty conditions from the normal state.

Nowadays the IoT systems are widely used on several applications for wireless data transmission. Health care applications are coming to the top among the sector on employing IoT for data transmission. An experiment was conducted to find the betterment among the 6LoWPAN and RPL IoT systems on health care data transmission [12]. The experiment was conducted by measuring the active parameters from an athletic person on their training process. The observations are kept to analyze the packet delivery ratio and routing protocol overhead among the different IoT systems. From the analysis it has been observed that the packet delivery ratio of 6LoWPAN achieves better performance than the RPL systems. However, the control packets delivered per node is comparatively larger in RPL over the 6LoWPAN module. 
Journal of Soft Computing Paradigm (JSCP) (2021)

Vol.03/ No.02

Pages: 96-109

http://irojournals.com/jscp/

DOI: https://doi.org/10.36548/jscp.2021.2.004

An elegant method for improving the performances of solar power system was developed with the help of IoT systems [13]. Here the system aims to monitor the sun light angle for tilting the solar panel angle to receive maximum radiation from the sun. The system also aims to identify the dust presence in the solar panels for analyzing the efficiency of the power generation. From the observation of dust particle presence the system developed to calculate the energy loss also. The dust particle observations will also create signal to clean up the dust presence in the solar panels. The smart grid architecture of IoT systems [14] are designed to observe multiple range of signals and parameters from the connected units. These kind of architecture improves connection and communication stability among the connected networks. The cost efficiency will also get improved while employing an IoT module with grid arrangement. Placing security systems on smart grid systems will minimize the effort of employing individual data security system for each sensors.

The IoT systems are also widely employed for improving the effective utilization of electrical energy. The challenges in employing solar power systems on highway lighting process are analyzed [15] to address its issues. The report says that the connected solar systems are in need of regular maintenance for improving the process efficiency. The challenge also explores that the identification of fault in such individual highway lighting system is difficult. In recent days for improving the IoT systems on network scalability and data mining process, the systems are equipped with a fog computing modules [16]. Hence the performances of IoT system will be improved to certain extent by means for estimating the optimum characteristics. Transmission of data from an IoT system requires very large space depending upon the application employed for. Saving and processing of such huge data will be difficult in a base station. Therefore to minimize the computational cost and energy the fog and cloud computing strategies are developed.

A big data mining technique was employed in a micro grid solar power system for detecting its performance efficiency [17]. From the analysis work it has been observed that the efficiency of solar power systems are fluctuated due to line resistance and poor application stability. The stability also has direct relationship over the load fluctuations on different scenarios. For addressing such issues the system is employed with a control discrete algorithm for analyzing the observed data from the connected systems. Keeping the collected information 
Journal of Soft Computing Paradigm (JSCP) (2021)

Vol.03/ No.02

Pages: 96-109

http://irojournals.com/jscp/

DOI: https://doi.org/10.36548/jscp.2021.2.004

on cloud and other public storage for mining process is not a good thing to certain extent. Because there's a possibility that such data will be tampered with and altered by hackers. This may lead to minor and major challenges in the connected systems of IoT architecture. In order to address such kind of challenges, several algorithms were developed. An effective perturbation algorithm was developed to measure the soil nutrients for analyzing the potential productivity tests [18]. The developed algorithm was verified with several datasets for identifying its prediction accuracy and attack resistance process.

A hybrid algorithm was also developed to estimate energy peak load and energy demand analysis on an electric energy systems [19]. The analysis was performed here with the help of support vector regression to identify the energy demand and peak load analysis. The observed performance analysis from support vector regression is also forwarded to particle swarm optimization for the value optimization. The analyzed information from the data mining algorithms on IoT sensor values are kept under the blockchain network for the future analysis [20]. As a result, the information from previous analyses may be retraced at any moment. Instead of storing the acquired data in the physical layer, it's a better idea to maintain it on the cloud, where it may be secured using blockchain technology. The data available in the blockchain can also be traced easily at any time for the future mining process.

\section{Proposed Work}

The block diagram of the proposed model is shown in figure 2. Here, the solar power station is connected with an IoT hardware board for transferring the observed signals to cloud environment for the data mining process. In data mining process, the performance of the solar generated power is compared to its previously generated power for the same sun light radiation. If the algorithm detects any deviation from the observed power generation values to the previous generated value means, the proposed model will send a signal to the maintenance team for an action. 


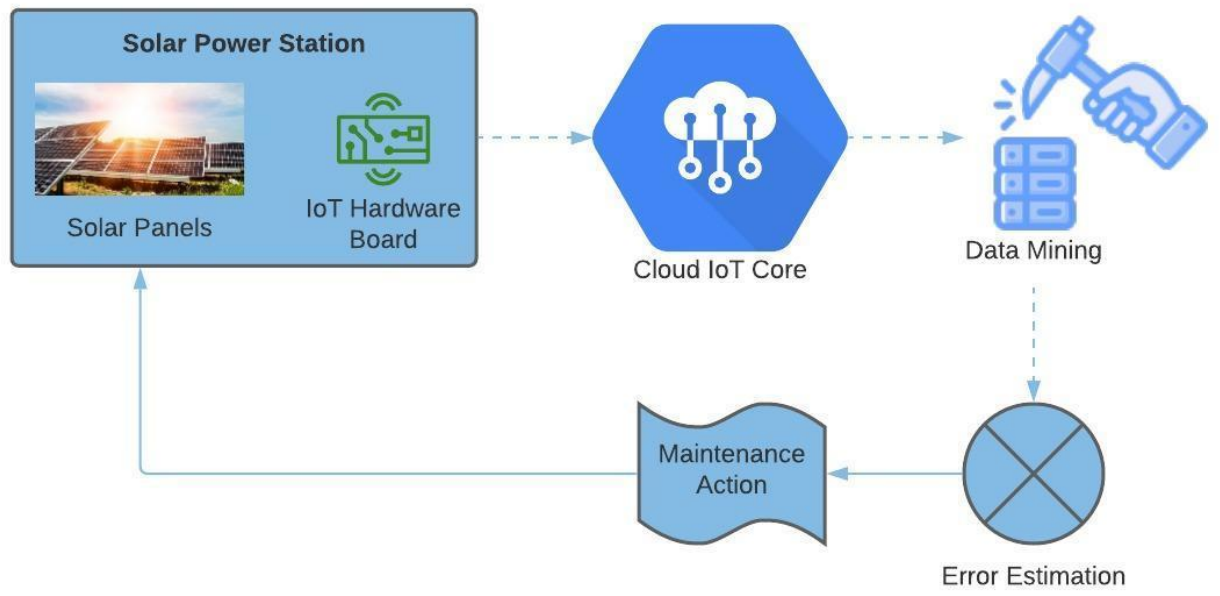

Figure 2. Block Diagram of the Proposed Model

The solar panel used for power generation is connected with a voltage and current sensor for measuring the generated output. Similarly, to measure the sunlight intensity, a set of LDR and photodiode was utilized in the proposed model. The connection setup has been established between the solar power station and microcontroller unit as shown in figure 3 . The observed signals from the sensor are connected into a signal conditioning circuit for removing the unwanted and interrupted signals. The sensors are usually created to produce analog signals in nature. Therefore, the fluctuations in the generated signal can be easily found by the microcontroller module.

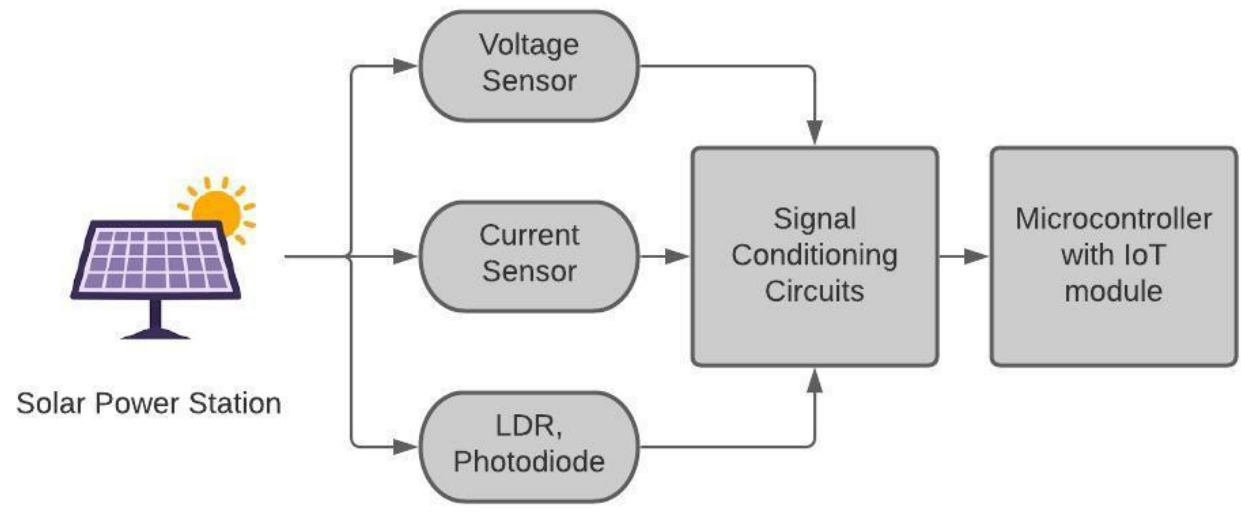

Figure 3. Connection between solar power stating and IoT module

Figure 4 explores the algorithmic flow of the proposed model. Here the sensors are connected to the solar panel for monitoring the generated power continuously. The observed 
Journal of Soft Computing Paradigm (JSCP) (2021)

Vol.03/ No.02

Pages: 96-109

http://irojournals.com/jscp/

DOI: https://doi.org/10.36548/jscp.2021.2.004

values are compared to the calibrated value of the connected solar panels. All the electrical equipment needs calibrations after the usage of certain time limit to verify its efficiency. In the proposed work those calibrated values are taken into account for the data mining process. To monitor the current and voltage variations thingsboard IoT platform is employed in the work. It will assist the maintenance team to monitor the virtual response of the connected solar power system. Simultaneously the same data is transmitted to a local microcontroller for analyzing the received value to the actual calibrated value. The actual value is kept over the microcontroller program at the time of every calibration cycle.

Solar panel crack, abnormal dust over the solar panels and poor tilting angle direction of solar panel are some of the causes for getting reduced and irregular power generation from the solar power systems. Nowadays there are lot of automated systems are developed to address these issues by means of image processing algorithms [21, 22] and machine learning algorithms $[23,24]$. The proposed system can also clubbed with such automated systems for improving their efficiency.

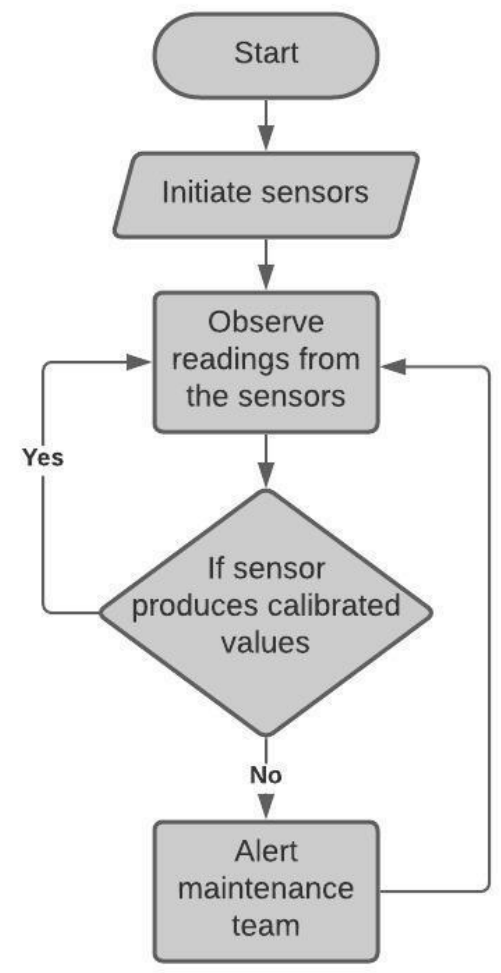

Figure 4. Workflow of the proposed model

ISSN: 2582-2640 (online) 
Journal of Soft Computing Paradigm (JSCP) (2021)

Vol.03/ No.02

Pages: 96-109

http://irojournals.com/jscp/

DOI: https://doi.org/10.36548/jscp.2021.2.004

\section{Experimental Analysis}

The proposed work is verified with two set of solar panel groups which are connected in series of 6 solar panels in a row to generate the maximum power of 2100 watts. Each solar panel has the ability to generate 350 watts on its maximum. The two solar panel groups are specified as solar panel $\mathrm{A}$ and $\mathrm{B}$ in figure 5 which are showing the output voltage, current and power from the generated from both of the solar panel group A and B. The utilized IoT platform has the ability to project the observed variations on a real time display with a given time interval. In the same way the temperature, LDR and photodiode outputs are projected in figure 6.

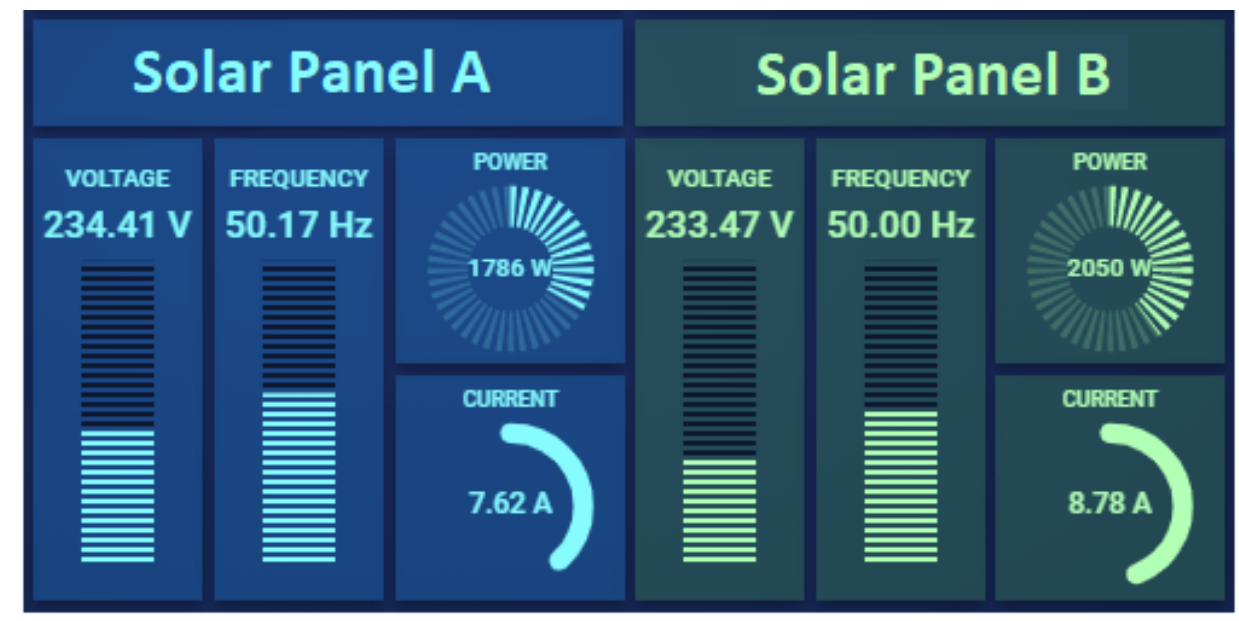

Figure 5. Output of the connected panels in thingsboard platform

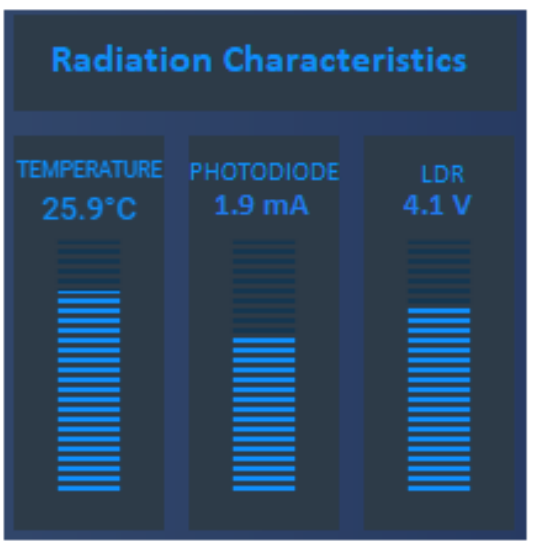

Figure 6. Temperature, photodiode and LDR output

ISSN: 2582-2640 (online) 
Journal of Soft Computing Paradigm (JSCP) (2021)

Vol.03/ No.02

Pages: 96-109

http://irojournals.com/jscp/

DOI: https://doi.org/10.36548/jscp.2021.2.004

The voltage and current fluctuations among the connected solar panel group A and B is shown in figure 7 and 8 . The display panels are also showing the minimum and maximum values of the measured parameters along with their average value on such specific time interval. In order to generate the alert signal, the microcontroller unit placed at the base station will be connected with a siren and LED display to project the status of the connected system. The trigger values are carried into account for analysis based on the values given to the microcontroller system from the calibrated values of the connected solar panels.

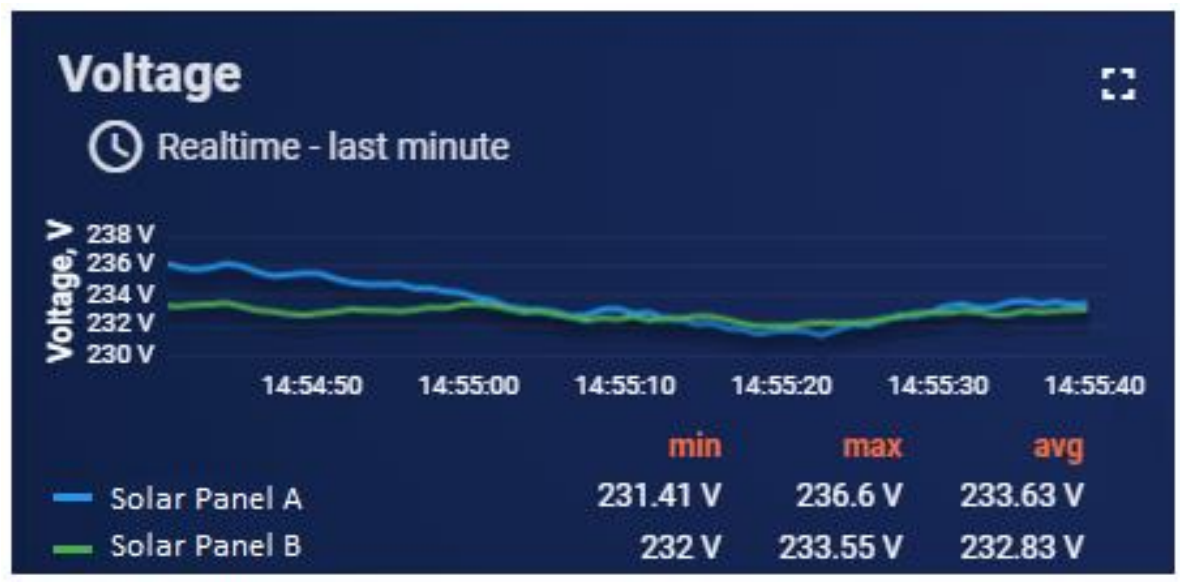

Figure 7. Realtime voltage variations

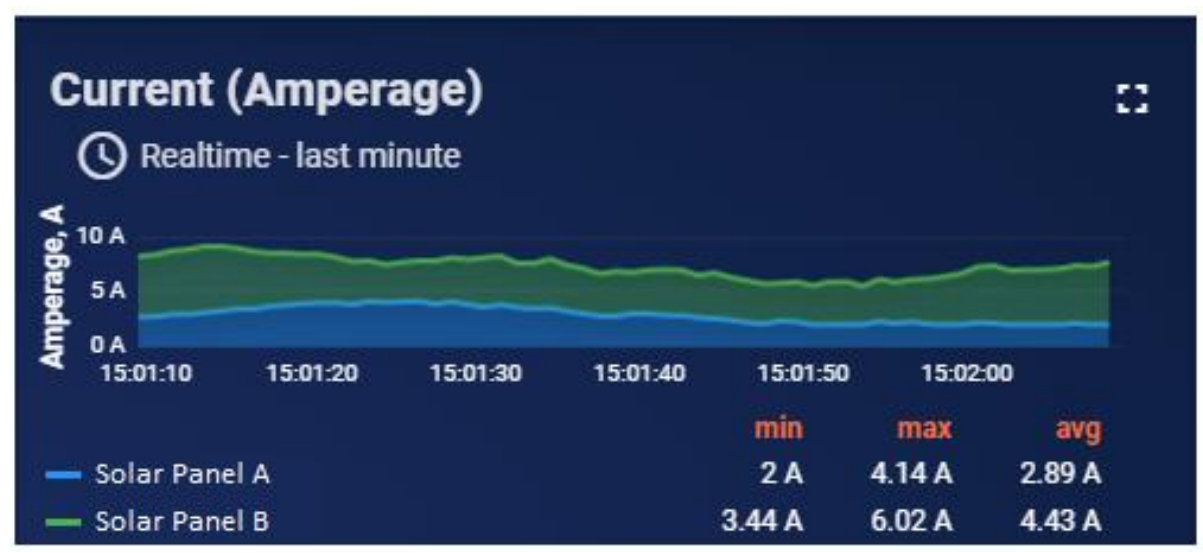

Figure 8. Realtime current variations

The projections from the IoT platform indicate that, the proposed work has the ability to efficiently monitor the performance of the connected solar panels. In future, the proposed work 
Journal of Soft Computing Paradigm (JSCP) (2021)

Vol.03/ No.02

Pages: 96-109

http://irojournals.com/jscp/

DOI: https://doi.org/10.36548/jscp.2021.2.004

can be connected with a vice versa communication model for sending back the control and operation signals for automating the process of solar panel cleaning and angle change direction process. To make such kind of automatic system in a reliable manner, the systems are required to be connected with an uninterrupted communication channel.

\section{Conclusion}

Solar power systems are the type of renewable power generation energy source, which are widely employed on domestic and commercial segments due to their simple construction. However, the solar power system is the one which requires huge maintenance for establishing its efficient operation. The work proposed in the paper aims to provide a maintenance alert based on the generated current and voltage from the solar panels. The observations from the solar panel systems are compared with respect to the calibrated values of the solar panel based on different solar radiations. The proposed model alerts the maintenance team, when there is a huge variation in the power generated by the solar panels. The proposed work can be extended with a control unit for performing some of the minor maintenance work on the connected solar panels.

\section{References}

[1] Prasad, B. Hari, and Mahesh Bhardwaj. "An Integrated Maintenance Management: A Practical Approach." In Recent Advances in Mechanical Engineering, pp. 141-147. Springer, Singapore, 2021.

[2] Amanuel, Thomas, Amanuel Ghirmay, Huruy Ghebremeskel, Robel Ghebrehiwet, and Weldekidan Bahlibi. "Design of Vibration Frequency Method with Fine-Tuned Factor for Fault Detection of Three Phase Induction Motor." Journal of Innovative Image Processing (JIIP) 3, no. 01 (2021): 52-65.

[3] van Staden, Heletjé E., and Robert N. Boute. "The effect of multi-sensor data on conditionbased maintenance policies." European Journal of Operational Research 290, no. 2 (2021): 585-600.

[4] Tesfamikael, Hadish Habte, Adam Fray, Israel Mengsteab, Adonay Semere, and Zebib Amanuel. "Construction of Mathematical Model of DC Servo Motor Mechanism with PID 
Journal of Soft Computing Paradigm (JSCP) (2021)

Vol.03/ No.02

Pages: 96-109

http://irojournals.com/jscp/

DOI: https://doi.org/10.36548/jscp.2021.2.004

controller for Electric Wheel Chair Arrangement." Journal of Electronics 3, no. 01 (2021): 49-60.

[5] Murugan, Raji, and Ramasamy Raju. "Evaluation of in-service power transformer health condition for Inspection, Repair, and Replacement (IRR) maintenance planning in electric utilities." International Journal of System Assurance Engineering and Management 12, no. 2 (2021): 318-336.

[6] Amanuel, Thomas, Amanuel Ghirmay, Huruy Ghebremeskel, Robel Ghebrehiwet, and Weldekidan Bahlibi. "Comparative Analysis of Signal Processing Techniques for Fault Detection in Three Phase Induction Motor." Journal of Electronics 3, no. 01 (2021): 61-76.

[7] Gonzalo, Alfredo Peinado, Alberto Pliego Marugán, and Fausto Pedro García Márquez. "Survey of maintenance management for photovoltaic power systems." Renewable and Sustainable Energy Reviews 134 (2020): 110347.

[8] Bhalaji, N., and C. Rimi. "Remaining Useful Life (RUL) estimation of lead acid battery using bayesian approach." J. Electr. Eng. Autom 2 (2020): 25-34.

[9] Li, Sai, Huajing Fang, and Bing Shi. "Remaining useful life estimation of lithium-ion battery based on interacting multiple model particle filter and support vector regression." Reliability Engineering \& System Safety 210 (2021): 107542.

[10] Bindhu, V., and G. Ranganathan. "Effective Automatic Fault Detection in Transmission Lines by Hybrid Model of Authorization and Distance Calculation through Impedance Variation." Journal of Electronics 3, no. 01 (2021): 36-48.

[11] Jan, Sana Ullah, Young Doo Lee, and In Soo Koo. "A distributed sensor-fault detection and diagnosis framework using machine learning." Information Sciences 547 (2021): 777-796.

[12] Hariharakrishnan, Jayaram, and N. Bhalaji. "Adaptability Analysis of 6LoWPAN and RPL for Healthcare applications of Internet-of-Things." Journal of ISMAC 3, no. 02 (2021): 6981.

[13] Priyadharsini, K., Dinesh Kumar JR, A. Srikanth, V. Sounddar, and M. Senthamilselvan. "Elegant method to improve the efficiency of remotely located solar panels using IoT." Materials Today: Proceedings 45 (2021): 8094-8104.

[14] Mugunthan, S., and T. Vijayakumar. "Review on IoT based smart grid architecture implementations." j Electric Eng Autom 1, no. 1 (2019): 12-20. 
Journal of Soft Computing Paradigm (JSCP) (2021)

Vol.03/ No.02

Pages: 96-109

http://irojournals.com/jscp/

DOI: https://doi.org/10.36548/jscp.2021.2.004

[15] Mukta, Marufa Yeasmin, Md Arafatur Rahman, A. Taufiq Asyhari, and Md Zakirul Alam Bhuiyan. "IoT for energy efficient green highway lighting systems: Challenges and issues." Journal of Network and Computer Applications 158 (2020): 102575.

[16] Lai, Kong-Long, and Joy Iong Zong Chen. "Development of Smart Cities with Fog Computing and Internet of Things." Journal of Ubiquitous Computing and Communication Technologies (UCCT) 3, no. 01 (2021): 52-60.

[17] Tian, Peng, and Lin Zhang. "Big data mining based coordinated control discrete algorithm of independent micro grid with PV and energy." Microprocessors and Microsystems 82 (2021): 103808 .

[18] Koresh, Mr H. James Deva. "Analysis of Soil Nutrients based on Potential Productivity Tests with Balanced Minerals for Maize-Chickpea Crop." Journal of Electronics 3, no. 01 (2021): 23-35.

[19] Kazemzadeh, Mohammad-Rasool, Ali Amjadian, and Turaj Amraee. "A hybrid data mining driven algorithm for long term electric peak load and energy demand forecasting." Energy 204 (2020): 117948.

[20] Wang, Haoxiang. "IoT based Clinical Sensor Data Management and Transfer using Blockchain Technology." Journal of ISMAC 2, no. 03 (2020): 154-159.

[21] Chen, Haiyong, Huifang Zhao, Da Han, Weipeng Liu, Peng Chen, and Kun Liu. "Structureaware-based crack defect detection for multicrystalline solar cells." Measurement 151 (2020): 107170 .

[22] Adam, Edriss Eisa Babikir. "Survey on Medical Imaging of Electrical Impedance Tomography (EIT) by Variable Current Pattern Methods." Journal of ISMAC 3, no. 02 (2021): 82-95.

[23] Jebli, Imane, Fatima-Zahra Belouadha, Mohammed Issam Kabbaj, and Amine Tilioua. "Prediction of solar energy guided by pearson correlation using machine learning." Energy 224 (2021): 120109.

[24] Adam, Edriss Eisa Babikir. "Evaluation of Fingerprint Liveness Detection by Machine Learning Approach-A Systematic View." Journal of ISMAC 3, no. 01 (2021): 16-30. 
Journal of Soft Computing Paradigm (JSCP) (2021)

Vol.03/ No.02

Pages: 96-109

http://irojournals.com/jscp/

DOI: https://doi.org/10.36548/jscp.2021.2.004

\section{Authors Biography}

Subarna Shakya is currently a Professor of Computer Engineering, Department of Electronics and Computer Engineering, Central Campus, Institute of Engineering, Pulchowk, Tribhuvan University, Coordinator (IOE) , LEADER Project (Links in Europe and Asia for engineering,eDucation, Enterprise and Research exchanges), ERASMUS MUNDUS. He received $\mathrm{MSc}$ and $\mathrm{PhD}$ degrees in Computer Engineering from the Lviv Polytechnic National University, Ukraine, 1996 and 2000 respectively. His research area includes E-Government system, Computer Systems \& Simulation, Distributed \& Cloud computing, Software Engineering \& Information System, Computer Architecture, Information Security for EGovernment and Multimedia systems. 